\title{
Genomic analysis of SBP gene family in Saccharum spontaneum reveals their association with vegetative and reproductive development
}

Yanhui Liu ${ }^{1,2+}$, Mohammad Aslam ${ }^{1 \dagger}$, Li-Ang Yao ${ }^{2}$, Man Zhang ${ }^{2}$, Lulu Wang ${ }^{1}$, Huihuang Chen ${ }^{2}$, Youmei Huang ${ }^{2}$, Yuan Qin ${ }^{1,2^{*}}$ and Xiaoping Niu ${ }^{1 *}$

\begin{abstract}
Background: SQUAMOSA promoter binding proteins (SBPS) genes encode a family of plant-specific transcription factors involved in various growth and development processes, including flower and fruit development, leaf initiation, phase transition, and embryonic development. The SBP gene family has been identified and characterized in many species, but no systematic analysis of the SBP gene family has been carried out in sugarcane.

Results: In the present study, a total of 50 sequences for 30 SBP genes were identified by the genome-wide analysis and designated SSSBP1 to SSSBP30 based on their chromosomal distribution. According to the phylogenetic tree, gene structure and motif features, the SSSBP genes were classified into eight groups (I to VIII). By synteny analysis, 27 homologous gene pairs existed in SSSBP genes, and 37 orthologous gene pairs between sugarcane and sorghum were found. Expression analysis in different tissues, including vegetative and reproductive organs, showed differential expression patterns of SSSBP genes, indicating their functional diversity in the various developmental processes. Additionally, 22 SSSBP genes were predicted as the potential targets of miR156. The differential expression pattern of miR156 exhibited a negative correlation of transcription levels between miR156 and the SSSBP gene in different tissues.

Conclusions: The sugarcane genome possesses 30 SSSBP genes, and they shared similar gene structures and motif features in their subfamily. Based on the transcriptional and QRT-PCR analysis, most SSSBP genes were found to regulate the leaf initial and female reproductive development. The present study comprehensively and systematically analyzed SBP genes in sugarcane and provided a foundation for further studies on the functional characteristics of SSSBP genes during different development processes.
\end{abstract}

Keywords: Sugarcane, SBP genes, Phylogenetic analysis, Expression analysis

\footnotetext{
* Correspondence: yuanqin@fafu.edu.cn; xpniu0613@126.com

${ }^{\dagger}$ Yanhui Liu and Mohammad Aslam contributed equally to this work. 'Guangxi Key Laboratory of Sugarcane Biology, State Key Laboratory for Conservation and Utilization of Subtropical Agro-Bioresources, College of Agriculture, Guangxi University, Nanning 530004, China

Full list of author information is available at the end of the article
}

(c) The Author(s). 2021 Open Access This article is licensed under a Creative Commons Attribution 4.0 International License, which permits use, sharing, adaptation, distribution and reproduction in any medium or format, as long as you give appropriate credit to the original author(s) and the source, provide a link to the Creative Commons licence, and indicate if changes were made. The images or other third party material in this article are included in the article's Creative Commons licence, unless indicated otherwise in a credit line to the material. If material is not included in the article's Creative Commons licence and your intended use is not permitted by statutory regulation or exceeds the permitted use, you will need to obtain permission directly from the copyright holder. To view a copy of this licence, visit http://creativecommons.org/licenses/by/4.0/ The Creative Commons Public Domain Dedication waiver (http://creativecommons.org/publicdomain/zero/1.0/) applies to the data made available in this article, unless otherwise stated in a credit line to the data. 


\section{Background}

Various transcription factors have revealed their critical roles in organism-specific function by activating or suppressing the expression of target genes [1]. The SQUAMOSA promoter binding (like) proteins (SBPs/SPLs) represent a major family of plant-specific transcription factors. SBPs/SPLs proteins share a highly conserved 76 amino acids in length DNA binding domain, also known as SBP binding domain [2]. The first SBP/SPL protein was identified in Antirrhinum majus, and this protein could interact with the promoter sequence of the floral meristem gene SQUAMOSA [3]. As a multigene family, $S B P / S P L$ genes have been characterized from different species ranging from single-cell green algae to multicellular angiosperm $[4,5]$. There are $16 S B P / S P L$ genes identified in Arabidopsis [6], 19 in rice [7], and 41 in soybean [8]. SBP transcription factors play central roles in various aspects of plant development including $[2,9$, 10], flower development [11], leaf development [12], plant hormone signaling transduction [13], vegetative to reproductive phase transition $[14,15]$. For example, AtSPL3 participates in regulating flowering under long photoperiod, and constitutively expressed SPL3 shows early flowering [6]. AtSPL8 is a central regulator involved in the regulation of microsporogenesis and megasporogenesis. spl8 mutant shows pollen sac development defects, and overexpression SPL 8 affects plant fertility by GA-dependent signaling pathway [16]. Moreover, SPL8 and other $S P L$ genes influence gynoecium patterning through mediating auxin homeostasis [17]. In monocot plants, such as rice and maize, $S B P$ genes are also reported to modulate essential developmental processes. Overexpression of OsSPL14 during the reproductive stage significantly promotes panicle branching and increased grain yield [18]. OsSPL16 is also a regulator of grain size, shape, and quality [19]. OsSPL3 regulates crown root development [20]. For maize, SBP proteins encoding genes, unbranched 2 and unbranched3, affect plant architecture and yield traits by regulating the lateral primordia initiation [21].

Numerous studies have revealed that many development processes mediated by SBP proteins are closely related to miR156. It is reported that miR156 in Arabidopsis can complementarily bind to the 3' UTR of SPL3 mRNA, and reduce its expression level through translation repression or transcript cleavage $[10,11]$. In rice, overexpression of OsmiR156 decreased the expression of SPL genes, indicating the conserved interaction relationship between SPL and miR156 [22]. Similarly, miR156 targeted OsSPL16 and OsSPL13 control grain shape, size and quality in rice $[19,23]$. In switchgrass, miR156/SPL4 module controls aerial axillary bud formation and biomass yield [24].
Sugarcane (Saccharum spontaneum), one of the most economically valuable plant, is a perennial tropical or subtropical crop, contributing up to about $80 \%$ of sugar production and $40 \%$ biofuel feedstock in the world [25]. Since 2000 years ago, sugarcane has been cultivated as sugar crop in China and India [26]. This domesticated sugarcane cultivar is a cross between species $S$. officinarum and $S$. spontaneum and accounts for the major genome information to modern sugarcane cultivars [27]. Although the genome information of S. spontaneum L. is available [28], little progress has been made in sugarcane germplasm improvement through sexual propagation due to the degeneration of sugarcane reproductive organs [29]. Therefore, unveiling the fundamental mechanism of the sugarcane reproductive developmental process is necessary to develop improved varieties [30].

Concerning recent findings of SBPs roles in Arabidopsis, rice, and other plants, analysis of $S B P$ gene function in sugarcane will undoubtedly accelerate sugarcane germplasm improvement. In this present study, we systematically analyzed the SBP gene family of sugarcane for their gene structure, phylogeny, motif and domain composition, miR156 target site, and expression pattern in various tissues and organs. Besides, the interaction between the SBP genes and miR156 was critically examined to study their functional relationship during the reproductive stage in sugarcane.

\section{Results}

Identification and characterization of SBP genes in S. spontaneum

To identify of SBP genes in sugarcane, the HMM profile of the SBP domain was used as a query to search the sugarcane genome database and BLASTP program. Initially, 66 putative SBP proteins were identified from the sugarcane genome database. All the resulting sequences were further checked by SMART and pfam tools to confirm SBP domain. Sixteen proteins without SBP (CysCys-His-Cys, Zn2) motif or with incomplete SBP domain were removed. Finally, 50 SBP proteins were identified and used for further analysis. Among them, 13 SBP genes had 2, 3 or 4 alleles, including 7 SsSBPs with 2 allelic genes, 5 SsSBPs with 3 allelic genes and 1 SsSBP with 4 allelic genes. We named these SsSBPs as SsSBP1 to SSSBP30 based on their chromosomal locations and added -1 to -4 for their alleles (Table 1 ). To futher investigate the conserved status of the SBP domain, 30 SBP protein sequences from sugarcane were aligned to predict conserved domains. The alignment results showed that all SsSBP proteins contained the complete SBP domain and possessed the typical characteristics of SBP domain with two Zinc motifs (Zn1 and Zn2) and one nuclear localization signal (NLS) (Fig. 1). 
Table 1 The characteristics of identified SBP genes in sugarcane

\begin{tabular}{|c|c|c|c|c|c|c|c|c|c|c|}
\hline Transcript ID & Name & Chr & Genome locations & ORF & $\begin{array}{l}\text { Amino } \\
\text { acids }\end{array}$ & $\begin{array}{l}\text { MW } \\
(\mathrm{kDa})\end{array}$ & pl & GRAVY & Group & $\begin{array}{l}\text { Subcellular } \\
\text { localization }\end{array}$ \\
\hline Sspon.001A0040480 & SsSBP1-1 & $1 \mathrm{~A}$ & $108,429,474-108,435,971$ & 2904 & 967 & 105.6349 & 5.45 & -0.303 & $\|$ & Nuclear \\
\hline Sspon.001D0044800 & SsSBP1-2 & $1 \mathrm{D}$ & $109,076,291-109,081,860$ & 2697 & 898 & 98.0265 & 5.53 & -0.313 & $\|$ & Nuclear \\
\hline Sspon.002A0013930 & SsSBP2-1 & $2 \mathrm{~A}$ & $29,209,489-29,217,185$ & 1644 & 547 & 57.0766 & 9.25 & -0.244 & VII & Nuclear \\
\hline Sspon.002B0011930 & SSSBP2-2 & $2 B$ & $28,141,105-28,145,280$ & 1329 & 442 & 45.9953 & 6.53 & -0.343 & VII & Nuclear \\
\hline Sspon.002D0010972 & SsSBP2-3 & $2 \mathrm{D}$ & $23,780,730-23,784,862$ & 1227 & 408 & 42.4603 & 6.53 & -0.354 & VII & Nuclear \\
\hline Sspon.002A0015030 & SsSBP3-1 & $2 \mathrm{~A}$ & $31,009,987-31,013,034$ & 1179 & 392 & 40.9194 & 9.14 & -0.477 & VII & Nuclear \\
\hline Sspon.002B0012730 & SsSBP3-2 & $2 B$ & $29,525,208-29,528,451$ & 1191 & 396 & 41.2847 & 8.98 & -0.462 & VII & Nuclear \\
\hline Sspon.002C0016120 & SsSBP3-3 & $2 C$ & $35,044,657-35,076,244$ & 3195 & 1064 & 116.0996 & 7.94 & -0.351 & $\mathrm{VII}$ & Nuclear \\
\hline Sspon.002D0011720 & SsSBP3-4 & $2 \mathrm{D}$ & $25,045,205-25,048,503$ & 1173 & 390 & 40.7902 & 9.04 & -0.491 & VII & Nuclear \\
\hline Sspon.002B0008220 & SsSBP4-1 & $2 B$ & $20,690,286-20,695,232$ & 1269 & 422 & 42.7577 & 9.68 & -0.315 & $\mathrm{Vl}$ & membrane \\
\hline Sspon.002C0010600 & SsSBP4-2 & $2 C$ & $22,401,265-22,403,347$ & 576 & 191 & 19.3214 & 9.87 & -0.399 & $\mathrm{VI}$ & Nuclear \\
\hline Sspon.002D0015440 & SsSBP4-3 & $2 \mathrm{D}$ & $33,849,661-33,853,542$ & 723 & 240 & 24.4241 & 10.32 & -0.533 & $\mathrm{Vl}$ & Nuclear \\
\hline Sspon.002C0010571 & SsSBP5 & $2 C$ & $22,345,677-22,347,101$ & 570 & 189 & 19.3353 & 9.96 & -0.564 & $\mathrm{Vl}$ & Nuclear \\
\hline Sspon.002D0015430 & SsSBP6 & $2 \mathrm{D}$ & $33,845,362-33,846,340$ & 600 & 199 & 20.1822 & 9.96 & -0.512 & $\mathrm{Vl}$ & Nuclear \\
\hline Sspon.003A0000410 & SsSBP7-1 & $3 \mathrm{~A}$ & $1,071,412-1,075,547$ & 1185 & 394 & 41.3121 & 9.15 & -0.496 & VII & Nuclear \\
\hline Sspon.003B0003860 & SsSBP7-2 & $3 B$ & $7,790,347-7,793,952$ & 1494 & 497 & 52.3230 & 8.94 & -0.291 & VII & Nuclear \\
\hline Sspon.003C0005960 & SsSBP7-3 & $3 C$ & $11,433,782-11,437,627$ & 1446 & 481 & 51.5591 & 9.57 & -0.44 & VII & Nuclear \\
\hline Sspon.003A0019770 & SsSBP8 & $3 \mathrm{~A}$ & $50,439,706-50,445,244$ & 2550 & 849 & 94.3843 & 6.22 & -0.423 & IV & Nuclear \\
\hline Sspon.003C0027810 & SsSBP9 & $3 C$ & $70,928,512-70,931,044$ & 2604 & 867 & 96.6721 & 7.81 & -0.473 & IV & Nuclear \\
\hline Sspon.004A0021810 & SsSBP10-1 & $4 \mathrm{~A}$ & $62,618,391-62,622,079$ & 768 & 255 & 27.6747 & 8.78 & -0.693 & V & Nuclear \\
\hline Sspon.004B0022500 & SsSBP10-2 & $4 \mathrm{~B}$ & $66,878,517-66,882,065$ & 1152 & 383 & 41.8795 & 8.67 & -0.709 & V & Nuclear \\
\hline Sspon.004A0023470 & SsSBP11-1 & $4 \mathrm{~A}$ & $66,657,938-66,661,498$ & 1458 & 485 & 51.7285 & 9.09 & -0.557 & V & Nuclear \\
\hline Sspon.004D0025580 & SsSBP11-2 & $4 \mathrm{D}$ & $76,179,579-76,181,404$ & 1077 & 358 & 38.8064 & 9.36 & -0.673 & V & Nuclear \\
\hline Sspon.004A0023540 & SsSBP12 & $4 \mathrm{~A}$ & $66,840,410-66,841,321$ & 1422 & 473 & 50.3841 & 9.01 & -0.551 & V & membrane \\
\hline Sspon.004B0022100 & SsSBP13 & $4 \mathrm{~B}$ & $66,160,113-66,162,260$ & 894 & 297 & 31.0155 & 9.24 & -0.16 & III & Nuclear \\
\hline Sspon.004C0023650 & SsSBP14 & $4 C$ & $72,419,102-72,423,304$ & 1314 & 437 & 45.6945 & 7.63 & -0.296 & III & Nuclear \\
\hline Sspon.004D0023290 & SsSBP15-1 & $4 \mathrm{D}$ & $70,928,686-70,930,879$ & 936 & 311 & 32.8608 & 9.53 & -0.295 & III & Nuclear \\
\hline Sspon.008B0005752 & SsSBP15-2 & $8 \mathrm{~B}$ & $10,803,440-10,806,188$ & 1263 & 420 & 44.4747 & 9.22 & -0.588 & III & Nuclear \\
\hline Sspon.008C0006030 & SsSBP15-3 & $8 C$ & $13,386,438-13,389,568$ & 1284 & 427 & 45.1796 & 9.23 & -0.583 & III & Nuclear \\
\hline Sspon.004D0028460 & SsSBP16 & $4 \mathrm{D}$ & $82,589,080-82,591,351$ & 1260 & 419 & 45.7805 & 9.52 & -0.608 & V & Nuclear \\
\hline Sspon.005A0006871 & SsSBP17-1 & $5 A$ & $17,014,877-17,018,085$ & 1185 & 394 & 42.8134 & 7.47 & -0.643 & III & Nuclear \\
\hline Sspon.005D0002360 & SsSBP17-2 & $5 \mathrm{D}$ & $5,016,047-5,019,246$ & 1191 & 396 & 42.8995 & 7.47 & -0.641 & III & Nuclear \\
\hline Sspon.005A0007540 & SsSBP18-1 & $5 A$ & $18,447,549-18,450,470$ & 1203 & 400 & 40.9130 & 9.50 & -0.373 & VII & Nuclear \\
\hline Sspon.005C0003700 & SsSBP18-2 & $5 C$ & $9,553,547-9,557,033$ & 1104 & 367 & 37.6902 & 9.48 & -0.429 & $\mathrm{VII}$ & Nuclear \\
\hline Sspon.005D0002370 & SsSBP19 & $5 \mathrm{D}$ & $5,036,183-5,040,905$ & 1191 & 396 & 43.2850 & 7.42 & -0.716 & III & Nuclear \\
\hline Sspon.006A0002670 & SsSBP20-1 & $6 \mathrm{~A}$ & $6,325,351-6,330,604$ & 2856 & 951 & 104.3392 & 7.10 & -0.354 & $\|$ & Nuclear \\
\hline Sspon.006D0001140 & SsSBP20-2 & $6 \mathrm{D}$ & $3,638,437-3,642,110$ & 2298 & 765 & 84.0087 & 8.79 & -0.459 & $\|$ & Nuclear \\
\hline Sspon.006A0003761 & SsSBP21-1 & $6 \mathrm{~A}$ & $8,766,368-8,771,945$ & 1410 & 469 & 48.7223 & 8.15 & -0.456 & VII & Nuclear \\
\hline Sspon.006D0002450 & SsSBP21-2 & $6 \mathrm{D}$ & $6,732,739-6,738,087$ & 1419 & 472 & 49.8476 & 8.62 & -0.479 & VII & Nuclear \\
\hline Sspon.006A0018544 & SsSBP22 & $6 \mathrm{~A}$ & $71,945,300-71,953,194$ & 1065 & 354 & 37.6695 & 9.26 & -0.173 & VII & Nuclear \\
\hline Sspon.006A0019261 & SsSBP23 & $6 \mathrm{~A}$ & $75,415,532-75,423,459$ & 1005 & 334 & 35.1004 & 9.93 & -0.328 & VII & Nuclear \\
\hline Sspon.006B0001500 & SsSBP24-1 & $6 \mathrm{~B}$ & $3,848,048-3,852,283$ & 1248 & 415 & 43.1377 & 8.93 & -0.627 & VII & Nuclear \\
\hline Sspon.006C0001780 & SsSBP24-2 & $6 C$ & $4,165,730-4,169,347$ & 1251 & 416 & 42.8242 & 8.87 & -0.621 & VII & Nuclear \\
\hline
\end{tabular}


Table 1 The characteristics of identified SBP genes in sugarcane (Continued)

\begin{tabular}{|c|c|c|c|c|c|c|c|c|c|c|}
\hline Transcript ID & Name & Chr & Genome locations & ORF & $\begin{array}{l}\text { Amino } \\
\text { acids }\end{array}$ & $\begin{array}{l}\text { MW } \\
(\mathrm{kDa})\end{array}$ & $\mathrm{pl}$ & GRAVY & Group & $\begin{array}{l}\text { Subcellular } \\
\text { localization }\end{array}$ \\
\hline Sspon.006D0000820 & SsSBP24-3 & $6 \mathrm{D}$ & $2,918,125-2,922,066$ & 1236 & 411 & 42.5089 & 8.87 & -0.627 & VII & Nuclear \\
\hline Sspon.006C0002070 & SsSBP25 & $6 C$ & $4,855,835-4,856,954$ & 897 & 298 & 30.4877 & 9.80 & -0.63 & $\|$ & Nuclear \\
\hline Sspon.006D0002410 & SsSBP26 & $6 \mathrm{D}$ & $6,577,854-6,582,459$ & 726 & 241 & 26.0042 & 8.71 & -0.741 & VII & Nuclear \\
\hline Sspon.007A0010700 & SsSBP27 & $7 A$ & $22,289,512-22,304,260$ & 1998 & 665 & 72.2692 & 5.37 & -0.438 & । & Nuclear \\
\hline Sspon.007A0010740 & SsSBP28 & $7 \mathrm{~A}$ & $22,398,323-22,403,566$ & 1311 & 436 & 47.2836 & 5.56 & -0.463 & । & Nuclear \\
\hline Sspon.008B0001960 & SsSBP29 & $8 B$ & $2,868,989-2,873,212$ & 1272 & 423 & 45.4750 & 9.32 & -0.382 & V & Nuclear \\
\hline Sspon.ctg0104090 & SsSBP30 & tig00011976 & $14-4187$ & 1065 & 354 & 38.0538 & 9.30 & -0.512 & VII & Nuclear \\
\hline
\end{tabular}

The detailed information about the SsSBPs was deduced by ExPASy server, including protein length, molecular weight (MW), theoretical isoelectric point (pI) and the grand average of hydropathicity (GRAVY). The length of the SsSBPs ORF region varied from $570 \mathrm{bp}$ (SsSBP5) to $3195 \mathrm{bp}$ (SsSBP3-3) and the protein lengths ranged from 189 to 1064 amino acids. The MW of the proteins ranged from 19.3214 to $116.09958 \mathrm{kDa}$. The pI ranged from 5.37 to 10.32 , and the values of GRAVY were all negative, suggesting that all SsSBPs are hydrophilic. Moreover, the subcellular localization of $50 \mathrm{SsSBP}$ proteins was predicted by ProtComp software and found that all SsSBP proteins localized in the nucleus except SsSBP4 and SsSBP12 proteins, which have no NLS signal, and localize in the cell membrane (Fig. S1; Table 1).

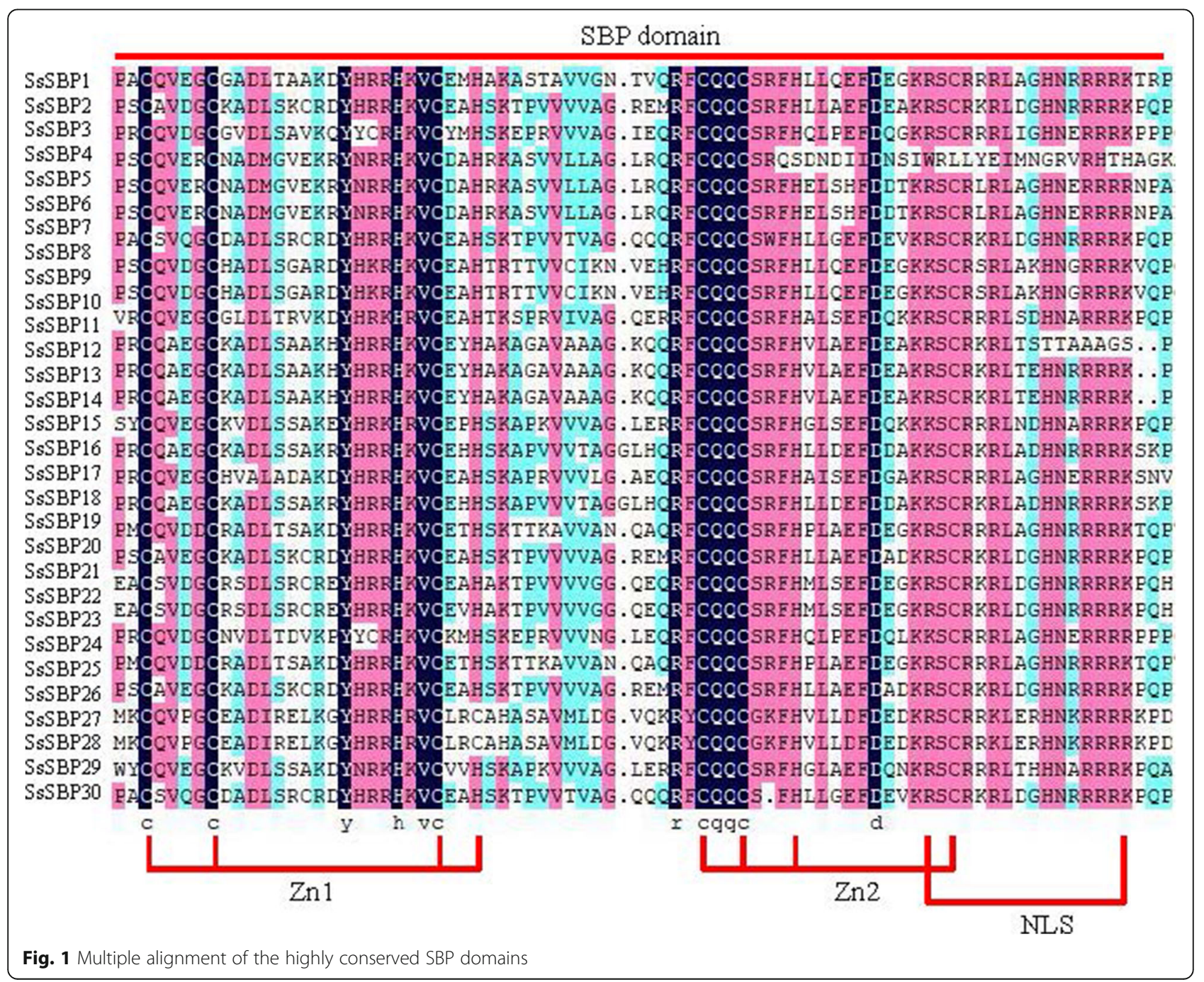




\section{Phylogenetic analysis of the SBP gene families}

We selected a total of 293 SBP homologs from 17 representative species from 7 green plant families, including chlorophytes, bryophytes, lycophytes, gymnosperms, basal magnoliophytes, eudicots and monocots, for phylogenetic analysis of SBP. Among them, both Ostreococcus sp. RCC809 and Ostreococcus Lucimarinus in green algae had only one SBP gene; $46 S B P$ genes exist in soybean. In comparison to the number of genes in these species, SBP genes in S. spontaneum showed an obvious expansion in the number of genes (Fig. 2). To gain further insight into the phylogenetic relationship of SSSBP genes, a phylogenetic tree was constructed using SBP proteins from Arabidopsis, Vitis vinifera, Ananas comosus, Sorghum bicolor and Oryza sativa (Fig. 3). SBP genes from these different species could be classified into 8 groups (I to VIII), and SBP proteins also tend to cluster the similar group. As expected, SsSBPs exhibited a closer relationship with the SBP proteins from S. bicolor and $O$. sativa. Group V and VII contained maximum SsSBP genes, where $S B P$ genes from $S$. bicolor and $O$. sativa were also grouped. While the group I contained only 2 members of $S S S B P$ genes formed the smallest group. This result was in agreement with the conservation analysis of the SBP proteins in other plants like Arabidopsis, grape, rice and sorghum. For example, a relatively high homologous genes, AtSPL6 / SbSBP5 / OsSPL1 / SsSBP8 / SSSBP9 clustered in one evolutionary branch (Fig. 3A). In addition, a ML phylogenetic tree was also constructed based on gene sequence similarity of 50 SsSBP proteins. The result indicated that the alleles of each SsSBP gene cluster in the same group, indicating that their sequences have high homology (Fig. 3B).

Structure characterization of SBP genes in S. spontaneum To better understand the genetic diversity of the SSSBP genes, the coding sequence of each gene was compared with their corresponding genomic sequence. The result revealed that the exon of SsSBP genes ranged from 2 to 13 in number (Fig. 4). SsSBP genes in group I contain 4-7 introns, group V contains 2-4 introns, group VI contains 1-2 introns, most of SsSBP members in group VIII contain 2 introns. Interestingly, alleles of $S S S B P$ genes $\quad(S S S B P 1-1 / 2, \quad$ SsSBP3-1/2/4, SsSBP4-1/2, SsSBP15-2/3, SsSBP17-1/2, SsSBP18-1/2, SsSBP20-1/2, SsSBP21-1/2 and SsSBP24-1/2/3) had the same number of exon/introns, although the length of introns varied. Some alleles possessed different exon/intron numbers (SsSBP2-1/2/3, SsSBP7-1/2/3, SsSBP10-1/2, SsSBP11$1 / 2$ ). The $S s S B P 3$ allele gene $S s S B P 3-3$ possessed the largest number (13) of exons, but the SsSBP3 alleles SsSBP3-1/2/4 only had 3 exons (Fig. 4). These results indicate that the number of exon and intron are diverse in different groups yet nearly consistent within the same group.

The SsSBP genes clustered into the same group exhibited similar structure and possessed a similar motif sequence. A total of 20 motifs were identified in SsSBP proteins, designated as motif 1-20 (Fig. 5, Fig. S2). The result expectedly showed that all SBP members contain Motif 1, Motif 2, Motif 3, Motif 5 and Motif 6, which was annotated as the SBP domain. Most of SBP

\begin{tabular}{|c|c|c|c|c|c|c|c|c|c|c|}
\hline \multirow{13}{*}{ Monocots } & Species & I & II & III & IV & $\mathrm{V}$ & VI & VII & VIII & Total \\
\hline & Brachypodium distachyon & 1 & 3 & 3 & 0 & 2 & 2 & 4 & 2 & 17 \\
\hline & Oryza sativa & 1 & 2 & 3 & 1 & 4 & 1 & 4 & 3 & 19 \\
\hline & Sorghum bicolor & 1 & 2 & 3 & 1 & 3 & 2 & 4 & 3 & 19 \\
\hline & Saccharum spontaneum & $2(2)$ & $3(5)$ & $5(8)$ & 2(2) & $5(7)$ & $3(5)$ & $7(12)$ & $3(9)$ & $30(50)$ \\
\hline & Ananas comosus & 0 & 5 & 4 & 1 & 1 & 0 & 3 & 2 & 16 \\
\hline & Populus trichocarpa & 2 & 6 & 2 & 3 & 5 & 5 & 4 & 1 & 28 \\
\hline & Glycine $\max$ & 2 & 8 & 4 & 6 & 2 & 10 & 6 & 8 & 46 \\
\hline & Arabidopsis lyrata & 1 & 4 & 1 & 1 & 3 & 2 & 1 & 2 & 15 \\
\hline & Arabidopsis thaliana & 1 & 4 & 1 & 1 & 3 & 3 & 1 & 2 & 16 \\
\hline & Vitis vinifera & 1 & 3 & 1 & 2 & 1 & 4 & 3 & 2 & 17 \\
\hline & Nymphaea colorata & 1 & 5 & 2 & 1 & 2 & 2 & 2 & 2 & 17 \\
\hline & Amborella trichopoda & 1 & 2 & 2 & 1 & 1 & 2 & 2 & 1 & 12 \\
\hline Lycophytes & Picea abies & 6 & 1 & 2 & 0 & 0 & 2 & 1 & 2 & 14 \\
\hline Bryophytes & Selaginella moellendorffii & 1 & 1 & 4 & 3 & 1 & 0 & 1 & 0 & 11 \\
\hline & Physcomitrella patens & 2 & 3 & 6 & 0 & 0 & 3 & 0 & 0 & 14 \\
\hline Chlorophytes & Ostreococcus lucimarinus & 1 & 0 & 0 & 0 & 0 & 0 & 0 & 0 & 1 \\
\hline & Ostreococcus sp. RCC809 & 1 & 0 & 0 & 0 & 0 & 0 & 0 & 0 & 1 \\
\hline & Total & 25 & 52 & 43 & 23 & 33 & 41 & 43 & 33 & 293 \\
\hline
\end{tabular}

Fig. 2 Distribution of SBP genes in 17 species 


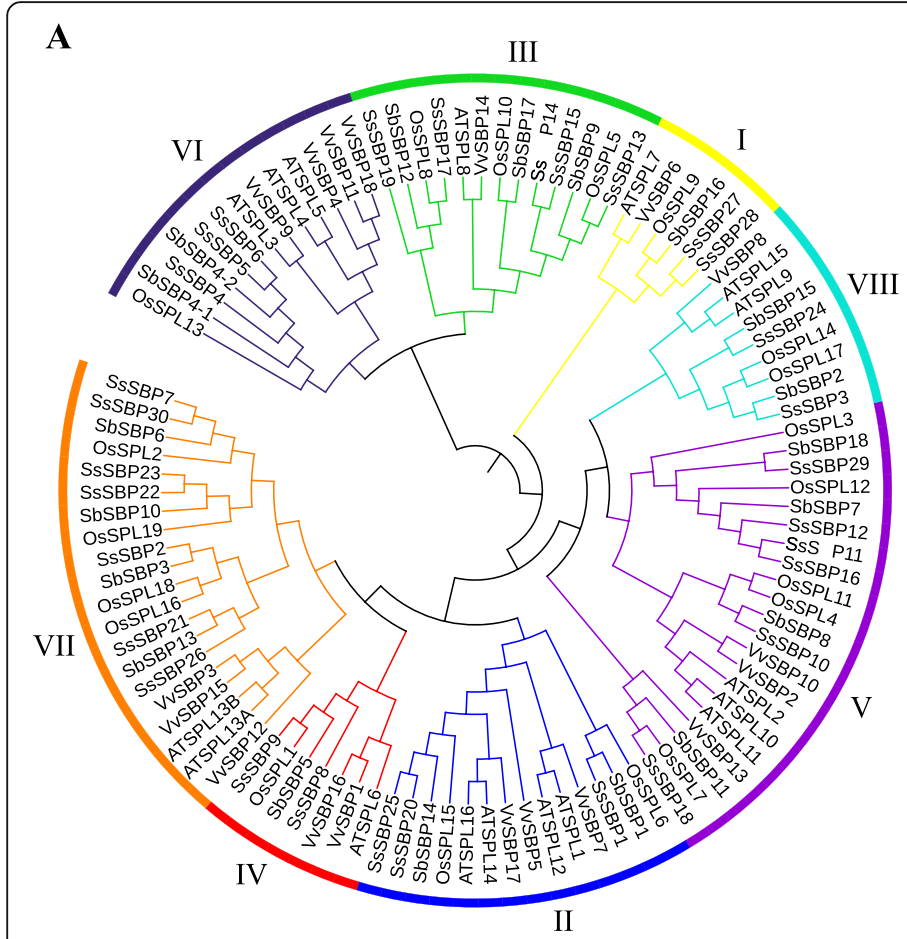

B

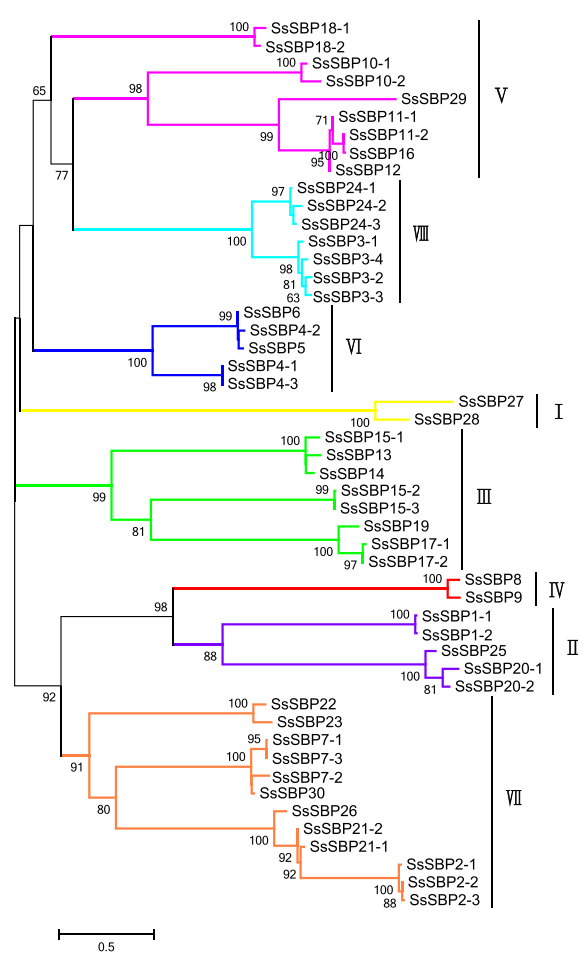

Fig. 3 Maximum likelihood phylogenetic tree of SBP gene family

members within the same group exhibited similar motif composition and exon-intron structure. In contrast, some motifs were found to be specific to one or two groups of SsSBP proteins. Motif 8 only appeared in group VIII. Motif 14, Motif 17 and Motif 19 were only present in group II, suggesting that proteins in these groups may have a specific biological function under a given condition. At the same time, the divergence among the different groups indicated their diverse functions.

\section{Chromosome distribution and gene duplication of SsSBP genes}

The chromosome distribution information of SSSBP genes revealed that 49 of the $50 S s S B P$ genes are located to the eight chromosomes of $S$. spontaneum, with the SsSBP30 mapped to the unanchored scaffolds (Fig. 6). On chromosomes 1 and 7, only two SsSBP genes were found. Chromosomes 3 and 5 contain five SsSBP genes. Chromosome 2 had the maximum number of $S S S B P$ genes with 12 members, followed by chromosome 6 with 11 SsSBP genes. In addition, 27 synteny gene pairs were identified in sugarcane using MCScanX software, with 24 pairs of alleles and 3 pairs of nonalleles. It should be defined as a tandem duplication event if a chromosomal region within 200 $\mathrm{kb}$ containing two or more genes [31]. According to this criterion, only two tandem duplications (SsSBP4-
3/SsSBP6 and SsSBP17-2/SsSBP19) were noticed (Fig. 6A, Table S3). These results indicate that segmental duplication events might significantly contribute to the SSSBP gene expansions than tandem duplication.

To further analyze the evolutionary process of SsSBP genes, a comparative analysis of genome synteny blocks between S. spontaneum and Sorghum bicolor was conducted. Sorghum is the closest related diploid to sugarcane, and the comparison of gene structures between these two species provided clues to the evolutionary gene events caused by polyploidization. A total of 37 syntenic gene pairs between $S$. spontaneum and $S$. bicolor were found (Fig. 6B, Table S3). To further understand the evolutionary forces on $S S S B P$ genes, the ratio of the synonymous $(K s)$ and nonsynonymous $(K a)$ substitutions rate $(K a / K s)$ was calculated for estimating the selection pressure of homologous genes, where $\mathrm{Ka} / \mathrm{Ks}<$ 1 indicates purifying selection, $K a / K s=1$ means neutral selection and $K a / K s>1$ indicates positive selection [32]. In this study, with the exception of three gene pairs SsSBP4-1/SsSBP5 (1.0379), SsSBP3-2/SsSBP3-4 (1.98497), SsSBP24-2/SsSBP24-3 (1.00893), Ka/Ks ratios of $S s S B P$ homologous genes were less than 1, indicating that these genes probably underwent a purifying selection (Table S2). Similarly, most $K a / K s$ values of sorghum genes were also less than 1, suggested that $S B P$ genes of these two close species underwent a strong purifying 


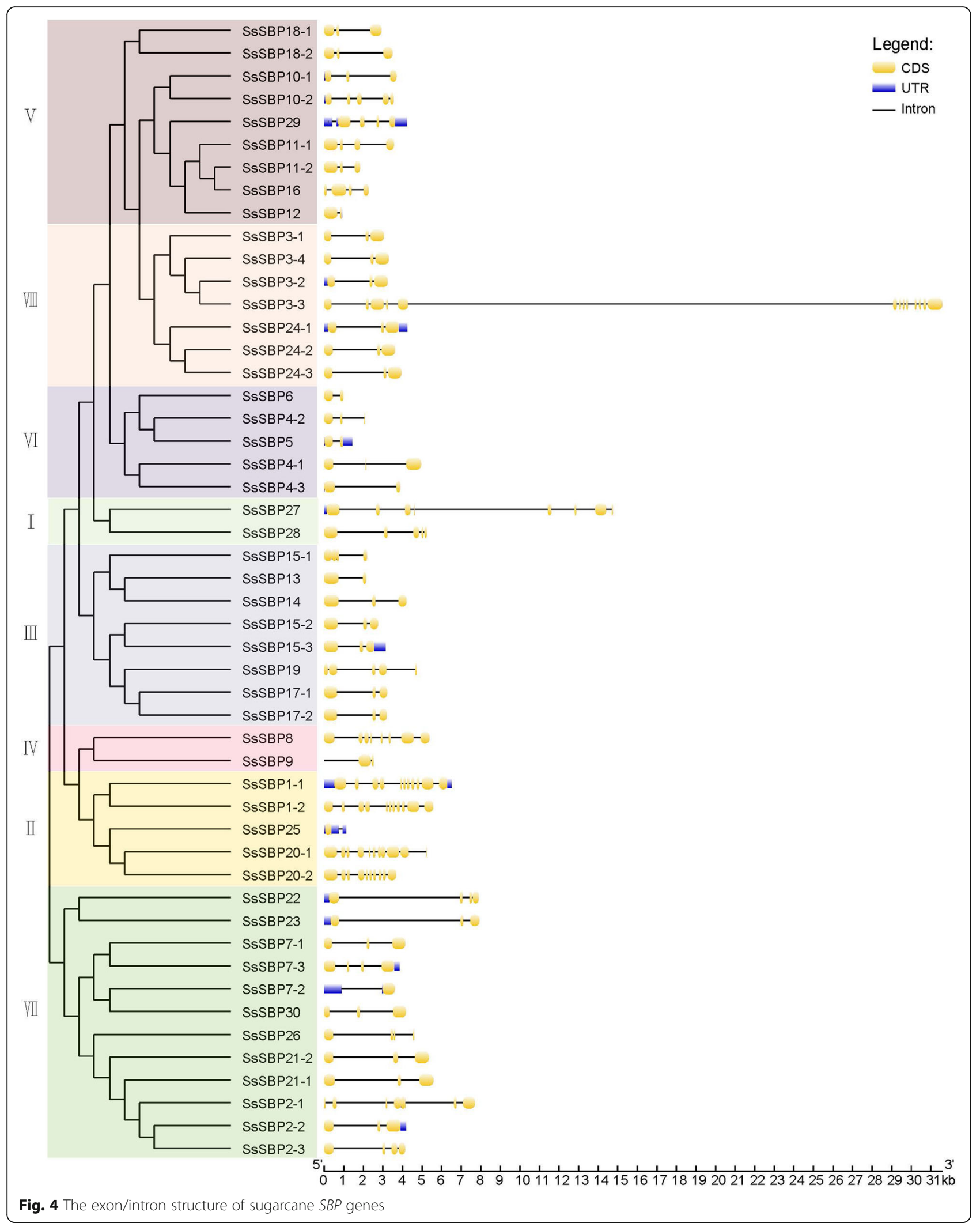




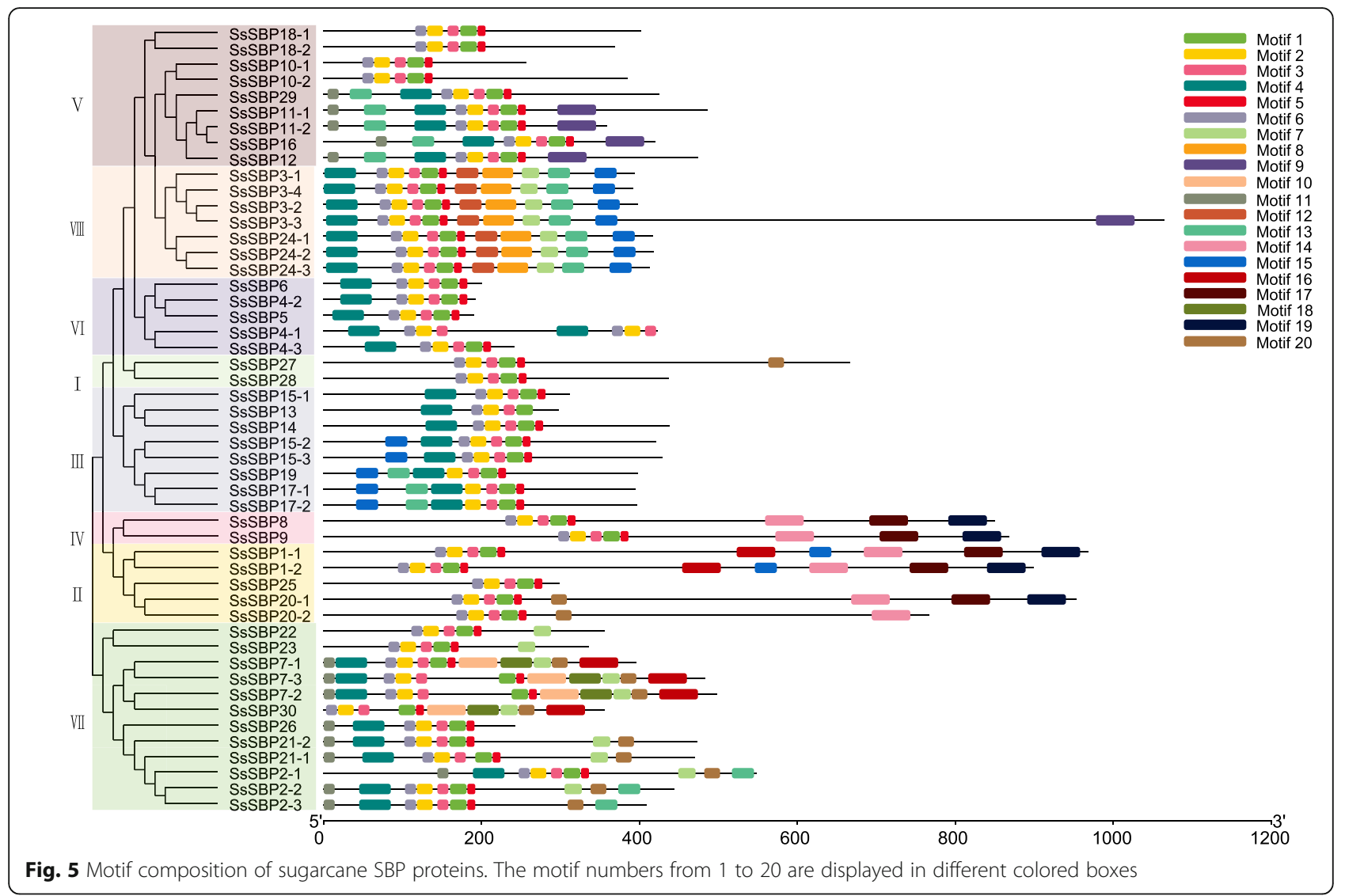

selection to reduce adverse mutations after duplication during the evolutionary process (Table S3).

\section{miR156 target prediction, distribution and expression pattern analysis}

Previous studies showed that miR156 complementarily binds to SBP genes either at the coding or 3'UTR region and reducs gene expression level through translation repression or transcript cleavage [11, 24]. In this present study, 22 SsSBP genes were found as the targets of miR156, and these genes were mainly distributed in groups V, VI, VII and VIII (Fig. 7A). Among these SsSBP genes, miR156 complementary sequences were at their coding regions except SsSBP5 where miR156 binding in the 3'-UTR (Fig. 7A; Fig. S3). Interestingly, the allelic genes SsSBP3-1, SsSBP3-2, SsSBP3-3 and SsSBP3-4 were all the targets of miR156 (Fig. 7A). Although there are 20 miRNA members of Saccharum sp., only one putative miRNA156 in S. spontaneum is represented in the miRbase database (https://www.mirbase.org/ v 22.1). Therefore, we performed a genome-wide study and found 29 members of miR156 genes in S. spontaneum genome. These Ssp-miR156 genes were mainly distributed on chromosomes $2,3,4,5,6,8$, except for chromosomes 1 and 7 (Fig. S4; Table S4). Ssp-miR156a, Ssp-
miR156f, Ssp-miR156j and Ssp-miR156k as alleles were localized on chromosome 2A. Chromosome 3A possesses 4 Ssp-miR156a alleles. Chromosome 3B contains 3 miR156 members (2 Ssp-miR156a and 1 Ssp-miR156l). Chromosome 4A has 4 miR156 members (3 SspmiR156a and 1 Ssp-miR156i), followed by 2 and 3 members on chromosome 4B (Ssp-miR156a and SspmiR156d) and 5A (2 Ssp-miR156a and 1 Ssp-miR156e), respectively. Two Ssp-miR156 were on chromosome 8A (Ssp-miR156e and Ssp-miR156k) and 8D (2 SspmiR156b). Only $1 S s p-m i R 156$ was found on chromosome 2C (Ssp-miR156b), 2D (Ssp-miR156b), 3C (SspmiR156a), 6A (Ssp-miR156a) and 6B (Ssp-miR156a). However, no Ssp-miR156 members was found on chromosomes 1 and 7 (Fig. S4; Table S4).

To further gain insight into the role of miR156 during female gametophyte development, we studied the miR156-SBP module during female gametophyte development. The results showed that the expression level of miR156 was mostly enriched in the mature stage of female reproductive development, and relatively low expression levels were found during the stages of $\mathrm{AC}$ (Archesporial Cell) to MMC (Megaspore Mother Cell). Generally, the expression level of miR156 increased from the initial stage to the mature stage of the female 

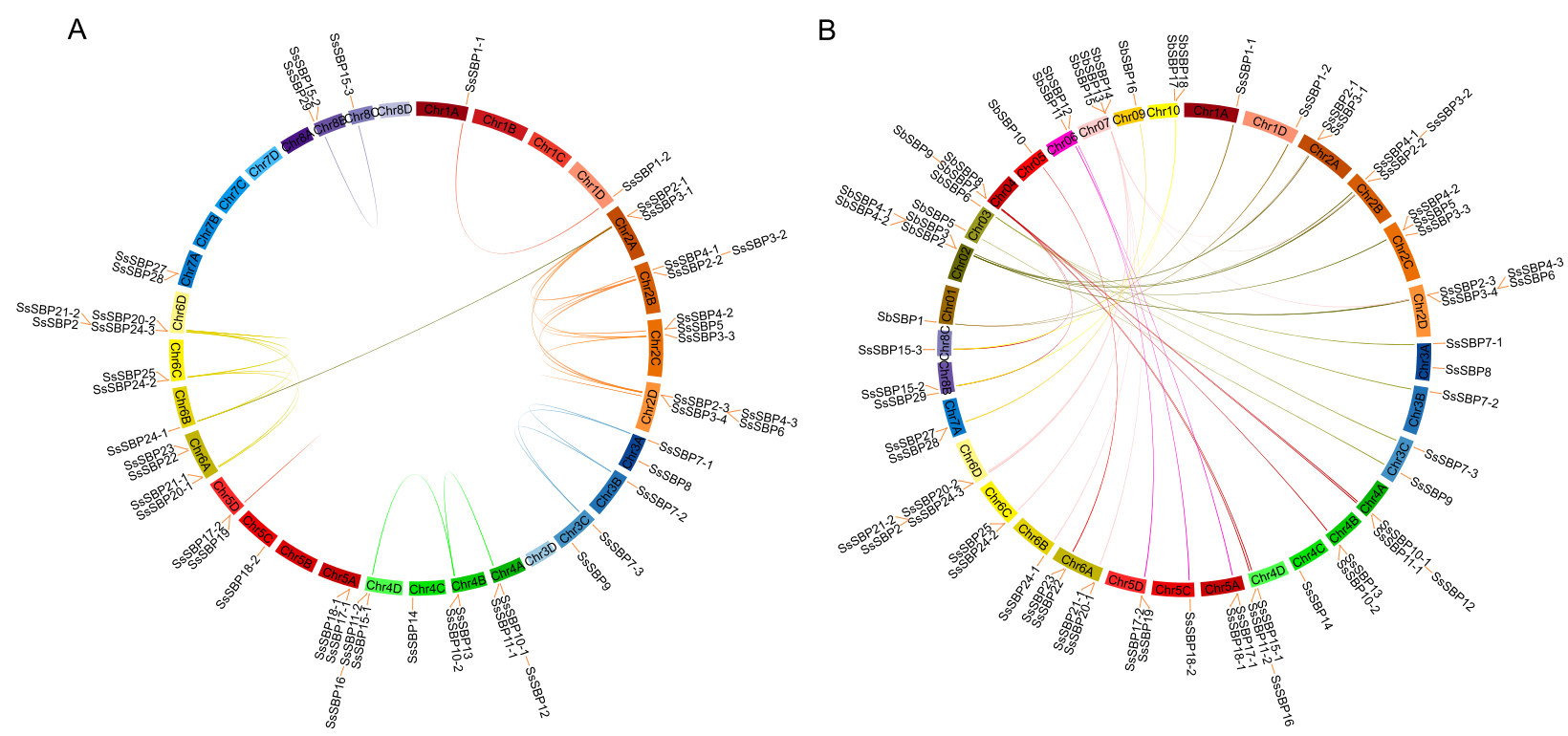

Fig. 6 Gene location and synteny in sugarcane. A Synteny analysis of SBP genes within sugarcane. B Synteny analysis of SBP genes between sugarcane and sorghum

gametophyte by sRNA-seq analysis (Fig. 7B; Table S5). In addition, the expression profiles of Ssp-miR156 precursors were also quantitatively verified using RT-PCR and qRT-PCR analysis. The results for the relative expression of Ssp-miR156 were consistent with the sRNAseq data (Fig. S5). On the contrary, the expression level of target $S S S B P$ genes was mostly decreased during the female gametophyte development stages, such as the target SsSBP11-1, SsSBP21-2, SsSBP22 and SsSBP30 (Fig. 7B).

To verify the authenticity of miR156-SBP module in sugarcane, we performed the degradome analysis and found that miR156 family members target the SsSBPs. The miR156k could bind the site $1400 \mathrm{bp}$ of its target SsSBP2-1 (Fig. 7C). Similarily, miR156a binds on the SsSBP3-2 (site 862) (Fig. 7D), SsSBP7-1 (site 850) (Fig. 7E), SsSBP7-3 (site 1110) (Fig. 7F), SsSBP10-1 (site 568) (Fig. 7G), SsSBP10-2 (site 1096) (Fig. 7H), SsSBP11-1 (site 1140) (Fig. 7I) and SsSBP21-1 (site 1065) (Fig. 7J). Taken together, these results suggest that miR156-SBP module is highly conserved, and the regulation pattern has diverged in different species.

\section{Expression profiles analysis of $S s S B P$ genes}

To study spatiotemporal expression patterns of SsSBP genes, RNA-seq data of different organs and tissues were analyzed. The expression level of SsSBP genes of leaf development and female reproductive organs is shown by heatmap representation (Fig. 8). As illustrated in Fig. 8A, SsSBP4, SsSBP6, SsSBP13, SsSBP14, SsSBP18, SsSBP21 and $S s S B P 26$ sustained low expression level in sugarcane leaf gradient segments, while SsSBP1 and SsSBP20 showed high expression in the leaf gradient segments. The transcript levels of SsSBP7, SsSBP10, SsSBP19, SsSBP22, SsSBP28 and SsBP29 decreased gradually from base to mature zone of leaf in sugarcane, showed that gene expression decreased following the maturing leaf (Fig. 8A, Table S6).

To investigate the SsSBP genes involvement in sugarcane female reproductive organ development, the transcription level of all SsSBP genes was extracted from RNA-seq data of sugarcane female reproductive organs. The heat map represents expression levels in the lines at five developmental stages shown in Fig. 8B. Many SsSBP genes showed different expression patterns among these five development stages. SsSBP1 and SsSBP10 were highly expressed in different stages of female gametophyte development. The transcripts of 7 SsSBP genes (SsSBP13, SsSBP14, SsSBP15, SsSBP16, SsSBP17, SsSBP18, SsSBP19) were zero in all these samples. The expression level of SsSBP7 and SsSBP30 showed differential expression during the female gametophyte development, revealing that these two genes may play an important role in $\mathrm{AC}$ and MMC stages (Fig. 8B, Table S7).

We also performed qRT-PCR experiments to confirm the expression level of some $S s S B P$ genes in those different female developmental stages. As shown in Fig. S6, the results of qRT-PCR data were highly consistent with the RNA-seq data for the relative expression of SsSBP genes during the female gametophyte development. Further studies may focus on the role of these genes on female reproductive development. 

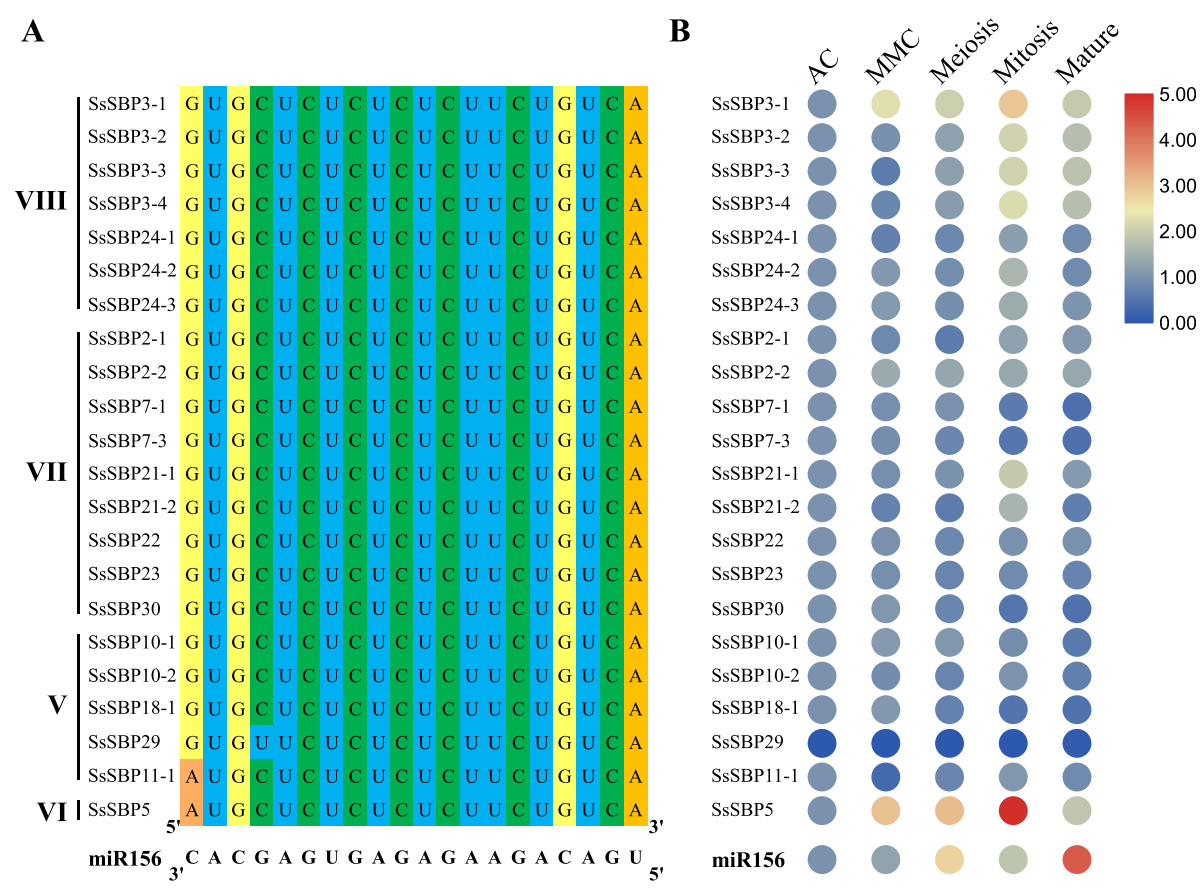

C

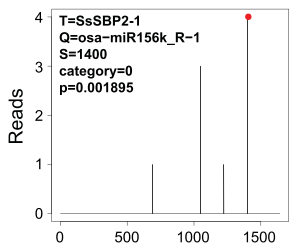

G

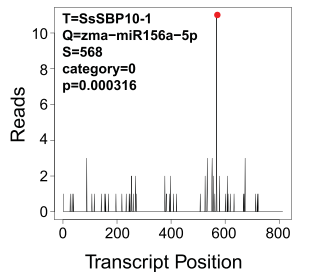

D

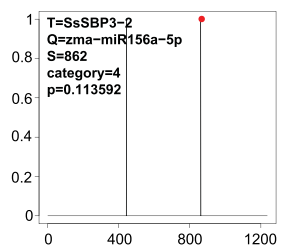

H

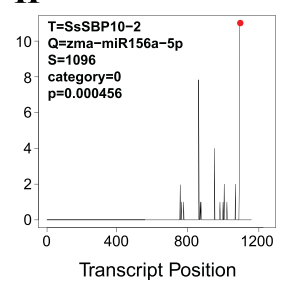

E

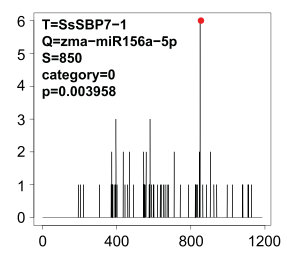

I

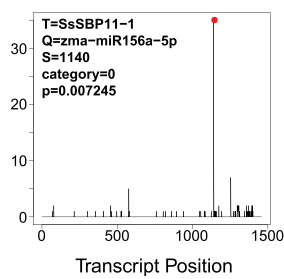

F

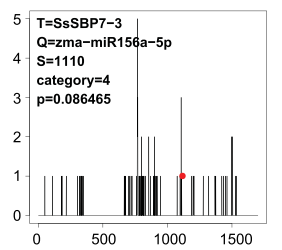

$\mathbf{J}$

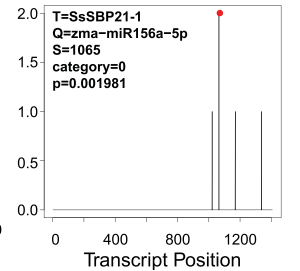

Fig. 7 Sequence alignment and expression patterns of miR156 compare with SsSBP genes. A Sequence alignment of the miR156 complementary sequences with the target sites in SSSBP genes. B The expression patterns of miR156 and their targets in different tissue samples. C-J The confirmation of miR156s and their targets by degradome ananlysis

\section{Discussion}

Sugarcane (S. spontaneum) has been widely domesticated and cultivated for thousands of years for its excellent economic values. It has become essential industrial material for sugar sources [25, 26]. The high quantity of genome data and abundance of increasing high-throughput transcriptome data make it possible to explore gene functions in non-model plants like Saccharum spp. Although the genome information of S. spontaneum L. is available, little progress has been made in sugarcane germplasm innovation and improvement due to the degeneration of sugarcane reproductive organs [28, 29]. Previous studies revealed that $S B P$ genes play crucial roles in plant development, especially in flower development, signaling transduction, and vegetative to reproductive phase transition [13-15]. However, the functions of $S$. spontaneum SBP genes remain unknown, although 17 SPLs were identified in sugarcane without taking alleles into account [33]. As for sugarcane genomic autopolyploidization, we conducted the genome-wide identification of SBP genes and their alleles in $S$. spontaneum, which resulted in the identification of 30 SBP genes (Fig. 1, Table 1). The number of SBP genes in $S$. spontaneum was similar to that in $P$. trichocarpa (28), O. zativa (19), and S. bicolor (19), but 


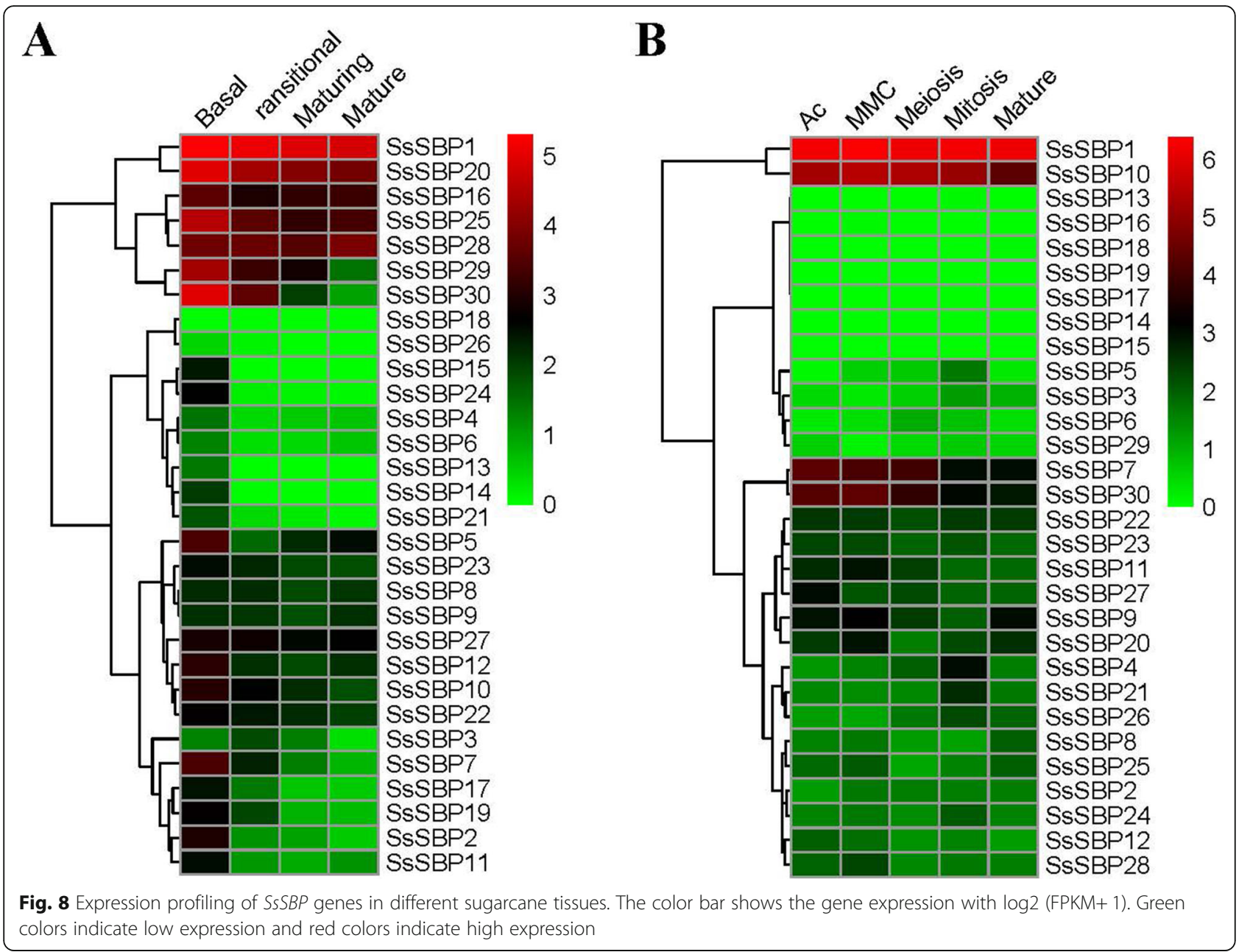

smaller than that in G. $\max$ (46), indicating that $S B P$ genes in different species underwent different gene duplication events. Based on phylogenetic and gene structure analysis, SSSBP genes could be divided into eight groups (group I-VIII), which is consistent with the results of previous studies on $S B P$ genes [34].

In general, the members of $S B P$ genes clustered into a subgroup shared similar gene structure and functions, suggesting these genes underwent common evolutionary origins. In other words, gene duplication events (segmental and tandem duplication) are the major driving forces for evolution and gene expansion by which many paralogous gene pairs are produced and could help organisms cope with different developmental processes [35]. In our study, a total of 27 duplication events were found in SSSBP genes, consisted of segmental duplications and tandem duplication (Fig. 6). The $\mathrm{Ka} / \mathrm{Ks}$ ratio is reported as the criterion for estimation the gene duplication. The $K a / K s$ ratio of a given $>1$ means that the gene has experienced positive selection, $=1$ suggests neutral selection and $<1$ indicates purifying selection. Based on the values of $K a / K s$ ratio, all the $S s S B P$ gene pairs were duplicated under purifying selection except gene pairs SsSBP4-1/SsSBP5 (1.0379), SsSBP3-2/SsSBP3-4 (1.98497), SsSBP24-2/SsSBP24-3 (1.00893) (Table S3). The diversity of SsSBP genes is likely to be motivated by gene duplication and genomic structure variation during the evolutionary process.

Up to now, there is little functional information on the SBP genes of sugarcane. Generally, the gene functions, to a large extent, are correlated to their expression patterns. In this present study, the expression levels of 30 SsSBP genes were examined across the four different leaf gradient segments and five female gametophyte development stages (Fig. 7). Most SsSBPS were predominantly expressed in the initial developmental stages of either leaf development or female gametophyte development. These results were similar with other species in the apical meristem, including apical buds inflorescences and flower buds $[9,10,22]$. Among the $S B P$ genes in Arabidopsis, AtSPL1 and AtSPL12 expressed highly in inflorescences and overexpression of these two genes enhanced the inflorescence thermotolerance [36]. AtSPL2, AtSPL9, AtSPL10, AtSPL11, AtSPL13 and AtSPL15 were reported 
to control the determination of leaf shape and the transformation of vegetative to reproductive stages [37]. Interestingly, the evolutionary analysis showed that AtSPL1 and AtSPL12 are highly orthologous to SsSBP genes in group II, including SsSBP1, SsSBP2O and SsSBP25. AtSPL2, AtSPL9 and AtSPL10 are orthologous to $S s S B P$ genes in the group V with $S s S B P 10$, SsSBP11, SsSBP12, SsSBP16, and SsSBP29. Based on the belief that homologous genes perform similar functions. SsSBP1 and SsSBP10, which were expressed highly in female gametophyte stages, would be involved in the development of female reproductive organs in sugarcane. Three genes SsSBP1, SsSBP20, SsSBP25 grouped with SsSBP16, SsSBP29 and SsSBP30, which are orthologous to AtSPL2, AtSPL9 and AtSPL10, expressed highly in the sugarcane leaves, confirming their roles in the regulation of leaf development. Certainly, additional studies need to be performed to confirm the potential roles in female gametophyte development (for SsSBP1 and SsSBP10) and leaf development (for SsSBP1, SsSBP20, SsSBP25, SsSBP16, SsSBP29 and SsSBP30).

In addition, miR156/SBP module has been reported to govern many aspects of plant growth and development [10, 17, 24, 38]. Overexpression of miR156 in Arabidopsis significantly repressed the SPL transcription and resulted in the loss of apical dominance, leading to dwarfism, an increase in total leaf number, and plant biomass [39]. Meanwhile, the expression levels of the target SBP genes of miR156 were suppressed in the miR156 overexpressing plants $[10,37]$. In the present study, the transcript level of miR156 was abundant in the mature stage of female reproductive development (Fig. 7B). In contrast, most putative target SsSBP genes predicted miR156 target sites showed lower expression levels in these tissues (Fig. 7B). These results suggested that the transcript of miR156 is negatively correlated with the expression of most SsSBP genes (Fig. 8). All together, our results revealed that miR156/SBP module could be used as an important tool to genetically improve crop architecture and productivity.

\section{Conclusion}

A total of $30 S B P$ genes were identified in sugarcane $(S$. spontaneum) by genome-wide analysis. These SsSBP genes were comprehensively characterized and classified into eight groups. The phylogenetic analysis showed that these genes shared orthologous relationships of SBP members from Arabidopsis and rice. The spatiotemporal expression patterns of these SsSBP genes in different tissues indicate that $S S S B P$ genes may regulate the leaf and female gametophyte development. Our results also showed that miR156 targeted many SsSBP genes. The expression level of miR156 was enriched in the female reproductive mature stages. The different expression levels between the miR156 and SsSBP genes in diverse tissues suggested that miR156/SBP module plays a crucial role in the leaf and female gametophyte development processes (Fig. 9). Taken together, our study provides the foundation for future in-depth elaboration of the potential functions of the $S B P$ genes in the growth and development of sugarcane.

\section{Methods}

\section{Identification and annotation of SBP genes in sugarcane}

Sugarcane genome data, CDS, protein sequence and annotation data were downloaded from the sugarcane Genome database (http://sugarcane.zhangjisenlab.cn/ sgd/html/index.html) [28]. Arabidopsis and other species sequences were searched and downloaded from Phytozome v13 (https://phytozome.jgi.doe.gov/pz/portal.html) [40]. To identify the SBP genes in sugarcane, the HMM profile of the SBP domain (PF03110) was downloaded from the Pfam database (http://pfam.xfam.org/) [41] and used as the query to search the sugarcane genome database. $S B P$ homologs were obtained by running a local BLASTP search using the Arabidopsis and rice SBP sequence as a query against the given protein database with an E-value cutoff of $10^{-5}$. The candidate genes were further confirmed by SMART server (http://smart. embl-heidelberg.de/). Sequences without the complete SBP domain were deleted. Finally, all the candidates were confirmed by multiple sequence alignments using DNAMAN software to ensure they contained the SBP domain. ExPASy (https://www.expasy.org/) [42] server was used to calculate the detailed information about the SsSBPs in sugarcane, such as molecular weights (MW), isoionic point ( $\mathrm{pI}$ ), and the grand average of hydropathicity (GRAVY). The subcellular localization of the SBP proteins identified was obtained using the ProtComp (v.9.0) software (http://www.softberry.com/).

\section{Gene structure, sequence alignments and phylogenetic analysis of SsSBP genes}

The exon/intron structure of $S B P$ genes was analyzed using Gene Structure Display Server (http://gsds.cbi.pku. edu.cn/index.php) [43] by comparing their coding and genomic sequences. Using BLASTP program to search homologous gene pairs among sugarcane and sorghum with the parameter of e-value $=1 \mathrm{e}-10$. The estimation of selection and substitution rates, the non-synonymous $(\mathrm{Ka})$, synonymous $(\mathrm{Ks})$ and $\mathrm{Ka} / \mathrm{Ks}$ substitution ratios of the homologous gene pairs of sugarcane and sorghum were calculated by the easy $\mathrm{Ka} / \mathrm{Ks}$ calculation program. MCScanX software [44] was used to detect the gene synteny and collinearity in sugarcane, and the SBPs locations were shown using Circos software [45]. Multiple sequence alignment of SBP protein sequence from Arabidopsis, rice, and sorghum was conducted using the 

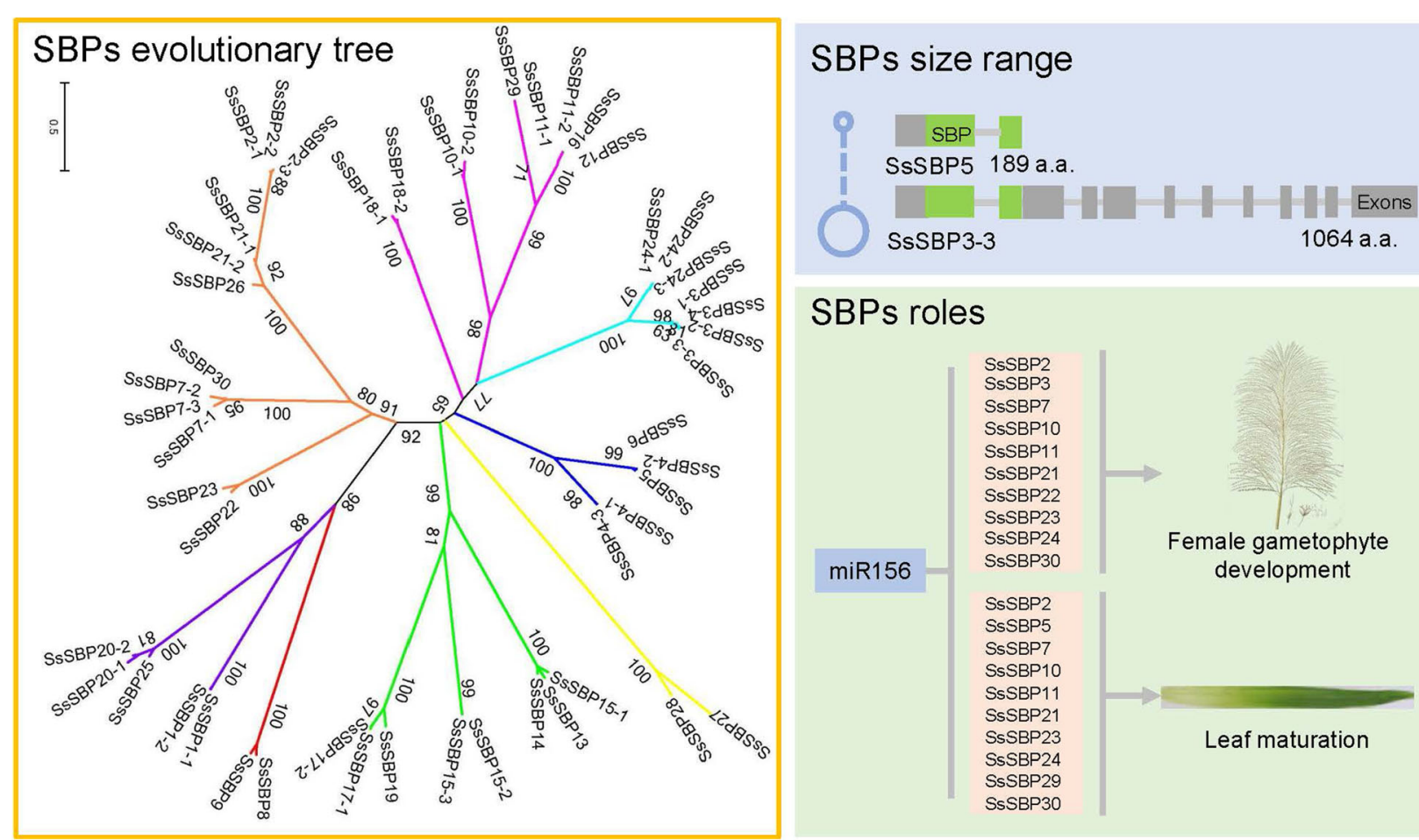

\section{SBPs roles}

Fig. 9 A summary work model of this study for SBP genes in sugarcane

MUSCLE in MEGA (v.6.0) [46]. A phylogenetic tree was constructed using RAxML software (http://www.phylo. org/index.php/) using the maximum likelihood (ML) method with bootstrap 1000 replications. The phylogenetic tree was displayed and manipulated using the Interactive tree of life (iTOL, https://itol.embl.de/) [47-49].

\section{Conserved motif identification, miR156 target site prediction and distribution}

The conserved motifs of SsSBP proteins were identified using the online program MEME (http://meme-suite. org/tools/meme) [50] with the default setting parameters: maximum number of motifs to find was 20; minimum width of motif was 6 and maximum width of motif was 50. The sequence logos of the SsSBP domain were showed by TBtools [51]. To predict the putative target sites of miR156, the cDNA sequences of SsSBP genes were analyzed using psRNATarget tool (http://plantgrn. noble.org/psRNATarget/). The chromosome location information of the Ssp-miR156s and SBPs were searched in sugarcane genome databases, and MapInspect software was used to generate chromosomal distribution information.

\section{Plant material and sample preparation}

The sugarcane (S. spontaneum L.) cultivar Yuetang 91976 was grown and collected by State Key Laboratory for Conservation and Utilization of Subtropical Agro-
Bioresources (Guangxi, China), and all samples from this cultivar was adopted for all experiment. When the plants reached the age of florescence stage, five different stages of the sugarcane female gametophyte development (i.e., AC, MMC, Meiosis, Mitosis and Mature) were collected. All samples were harvested as three biological replicates, which were quick-freeze with liquid nitrogen and stored at ultra-low temperature to facilitate the extraction of RNA.

RNA extraction, expression profiles and qRT-PCR analysis Total RNA was isolated by the Omega Total RNA kit II (R6934-02, USA). The evaluation of RNA quality was performed by the gel electrophoresis and 2000 spectrophotometer assessment at $260 \mathrm{~nm}$ (NanoDrop, Thermo Fisher Scientific), and Illumina sequencing was done using the method of Zhao et al. (2018).

For qRT-PCR analysis, the cDNA was synthesized using the ThermoScript RT-PCR kit (Life Technologies) in a $20 \mu \mathrm{L}$ volume reaction under the program: $42{ }^{\circ} \mathrm{C}$ for $15 \mathrm{~min}$ and $85^{\circ} \mathrm{C}$ for $15 \mathrm{~s}$. According to the SYBR Premix RT reagent kit system (TaKaRa, Dalian, China), the reaction contains $1 \mu \mathrm{g}$ RNA prior to qRT-PCR.

To understand the expression profiles of SBP genes, the RNA-seq data of leaf development were downloaded from the Saccharum Genome database (http:// sugarcane.zhangjisenlab.cn/sgd/html/index.html). The RNA-seq data of female reproductive development have 
been deposited in the European Nucleotide Archive (ENA, accession number PRJEB44944). Different leaf developmental stages, including basal zone, a transitional zone, a maturing zone, a mature zone $[52,53]$, and the female reproductive development stages, AC, MMC, Meiosis, Mitosis and Mature, were used for the study. The RNA-seq raw reads were filtered by Trimmomatic software with default parameters to obtain clean reads. The clean reads were mapped to the reference genome using Hisat2 [54]. Gene expression was calculated by Cufflinks software [55]. The log2-transformed RPKM value of the expression patterns of $S s S B P$ genes was used to generate the heatmap using the pheatmap package in $\mathrm{R}$ software. The expression pattern of miR156 was calculated by count values according to the miRNA-seq data.

To further confirm the expression profiles of the SsSBP genes, qRT-PCR assays were performed in different female reproductive development stages. qRT-PCR was conducted in CFX96 Real-Time System (Bio-Rad) using SYBR Green (TaKaRa) according to the instructions. Each reaction contains $12.5 \mu \mathrm{L}$ SYBR mixture, $1.0 \mu \mathrm{L}$ specific primer and $1 \mu \mathrm{g}$ sample template. Three replicate reactions were performed for each sample under the following program: $95^{\circ} \mathrm{C}$ for 30 s; 40 cycles of $95^{\circ} \mathrm{C}$ for $5 \mathrm{~s} ; 60^{\circ} \mathrm{C}$ for $30 \mathrm{~s}$. The primers used in this study are listed in Table S1.

\section{Supplementary Information}

The online version contains supplementary material available at https://doi. org/10.1186/s12864-021-08090-3.

\section{Additional file 1.}

\section{Acknowledgements}

We want to thank all the colleagues in our lab for providing valuable discussions and technical assistance. We are grateful to the reviewers for their critical and constructive comments on our manuscript.

\section{Authors' contributions}

$Y Q$ and $X N$ designed the research and wrote the manuscript. $Y L$ and $L W$ performed phylogenetic analysis and annotated the genes on chromosomes and conducted the evolution analysis. MA performed the sRNA-seq and degradome-seq. MA, MZ and YH analyzed data. LY, LW and $\mathrm{HC}$ performed qRT-PCR analysis. MA, XN and YQ revised the manuscript. All authors have read and approved the manuscript.

\section{Funding}

This work was supported by Science and Technology Major Project of Guangxi (Gui Ke 2018-266-Z01), National Natural Science Foundation of China (31800262; U1605212; 31761130074; 31600249; 31700279), China Postdoctoral Science Foundation (2018 M632564), Weng Hongwu Academic Innovation Research Fund of Peking University, and a Guangxi Distinguished Experts Fellowship.

\section{Availability of data and materials}

All data generated or analyzed during this study are included in this published article and its supplementary information files. The sequencing data of miRNA that support the findings of this study have been deposited in the NCBI SRA database with BioProject accession no. PRJNA723681 (https://dataview.ncbi.nlm.nih.gov/object/PRJNA723681? reviewer=vd8eph316 oqf79jo3ta52181 gr), and the RNA-seq data of female reproductive development have been deposited in the EMBL Nucleotide Sequence database (ENA) with accession no. PRJEB44944 (https://www.ebi.ac.uk/ena/ browser/view/PRJE44944), which will be available publicly upon acceptance of the article. The RNA-seq data of leaf development were downloaded from the Saccharum Genome database (http://sugarcane.zhangjisenlab.cn/sgd/ html/index.html).

\section{Declarations}

Ethics approval and consent to participate

The sugarcane (S. spontaneum L.) cultivar Yuetang 91-976 was grown and collected by State Key Laboratory for Conservation and Utilization of

Subtropical Agro-Bioresources (Guangxi, China), and all samples from this cultivar was adopted for all experiment. These plant materials don't include any wild species at risk of extinction. No specific permits are required for sample collection in this study. We comply with relevant institutional, national, and international guidelines and legislation for plant study.

\section{Consent for publication}

Not applicable.

\section{Competing interests}

The authors declare that they have no conflict of interest.

\section{Author details}

${ }^{1}$ Guangxi Key Laboratory of Sugarcane Biology, State Key Laboratory for Conservation and Utilization of Subtropical Agro-Bioresources, College of Agriculture, Guangxi University, Nanning 530004, China. ${ }^{2}$ College of Life Science, Fujian Provincial Key Laboratory of Haixia Applied Plant Systems Biology, Fujian Agriculture and Forestry University, Fuzhou 350002, China.

Received: 1 May 2021 Accepted: 15 October 2021

Published online: 27 October 2021

References

1. Gong W, Shen YP, Ma LG, Pan Y, Du YL, Wang DH, et al. Genome-wide ORFeome cloning and analysis of Arabidopsis transcription factor genes. Plant Physiol. 2004;135(2):773-82. https://doi.org/10.1104/pp.104.042176.

2. Yamasaki K, Kigawa T, Inoue M, Tateno M, Yamasaki T, Yabuki T, et al. A novel zinc-binding motif revealed by solution structures of DNA-binding domains of Arabidopsis SBP-family transcription factors. J Mol Biol. 2004; 337(1):49-63. https://doi.org/10.1016/j.jmb.2004.01.015.

3. Klein J, Saedler $H$, Huijser P. A new family of DNA binding proteins includes putative transcriptional regulators of the Antirrhinum majus floral meristem identity gene SQUAMOSA. Mol Gen Genet. 1996;250(1):7-16. https://doi. org/10.1007/BF02191820.

4. Riese M, Hohmann S, Saedler H, Munster T, Huijser P. Comparative analysis of the SBP-box gene families in P. patens and seed plants. Gene. 2007; 401(1-2):28-37. https://doi.org/10.1016/j.gene.2007.06.018.

5. Zhang SD, Ling LZ, Yi TS. Evolution and divergence of SBP-box genes in land plants. BMC Genomics. 2015;16(1):787. https://doi.org/10.1186/s12864015-1998-y.

6. Cardon GH, Hohmann S, Nettesheim K, Saedler H, Huijser P. Functional analysis of the Arabidopsis thaliana SBP-box gene SPL3: a novel gene involved in the floral transition. Plant J. 1997;12(2):367-77. https://doi.org/1 0.1046/j.1365-313X.1997.12020367.X

7. Yang Z, Wang X, Gu S, Hu Z, Xu H, Xu C. Comparative study of SBP-box gene family in Arabidopsis and rice. Gene. 2008;407(1-2):1-11. https://doi. org/10.1016/j.gene.2007.02.034

8. Tripathi RK, Goel R, Kumari S, Dahuja A. Genomic organization, phylogenetic comparison, and expression profiles of the SPL family genes and their regulation in soybean. Dev Genes Evol. 2017;227(2):101-19. https://doi.org/1 0.1007/s00427-017-0574-7.

9. Shikata M, Koyama T, Mitsuda N, Ohme-Takagi M. Arabidopsis SBP-box genes SPL10, SPL11 and SPL2 control morphological change in association with shoot maturation in the reproductive phase. Plant Cell Physiol. 2009; 50(12):2133-45. https://doi.org/10.1093/pcp/pcp148.

10. Wu G, Poethig RS. Temporal regulation of shoot development in Arabidopsis thaliana by miR156 and its target SPL3. Development. 2006; 133(18):3539-47. https://doi.org/10.1242/dev.02521. 
11. Gandikota M, Birkenbihl RP, Hohmann S, Cardon GH, Saedler H, Huijser P. The miRNA156/157 recognition element in the 3' UTR of the Arabidopsis SBP box gene SPL3 prevents early flowering by translational inhibition in seedlings. Plant J. 2007;49(4):683-93. https://doi.org/10.1111/j.1365-313X.2 006.02983.x.

12. Usami T, Horiguchi G, Yano S, Tsukaya H. The more and smaller cells mutants of Arabidopsis thaliana identify novel roles for SQUAMOSA PROMOTER BINDING PROTEIN-LIKE genes in the control of heteroblasty. Development. 2009;136(6):955-64. https://doi.org/10.1242/dev.028613.

13. Zhang Y, Schwarz S, Saedler H, Huijser P. SPL8, a local regulator in a subset of gibberellin-mediated developmental processes in Arabidopsis. Plant Mol Biol. 2007;63(3):429-39. https://doi.org/10.1007/s11103-006-9099-6.

14. Jung JH, Seo PJ, Kang SK, Park CM. miR172 signals are incorporated into the miR156 signaling pathway at the SPL3/4/5 genes in Arabidopsis developmental transitions. Plant Mol Biol. 2011;76(1-2):35-45. https://doi. org/10.1007/s11103-011-9759-z.

15. Yu S, Lian H, Wang JW. Plant developmental transitions: the role of microRNAs and sugars. Curr Opin Plant Biol. 2015;27:1-7. https://doi.org/10.1 016/j.pbi.2015.05.009

16. Unte, U. SJPC. SPL8, an SBP-box gene that affects pollen sac development in arabidopsis. Plant Cell. 2003;15(4):1009-19.

17. Xing S, Salinas M, Garcia-Molina A, Hohmann S, Berndtgen R, Huijser P. SPL8 and miR156-targeted SPL genes redundantly regulate Arabidopsis gynoecium differential patterning. Plant J. 2013;75(4):566-77. https://doi. org/10.1111/tpj.12221.

18. Miura K, Ikeda M, Matsubara A, Song XJ, Ito M, Asano K, et al. OsSPL14 promotes panicle branching and higher grain productivity in rice. Nat Genet. 2010;42(6):545-9. https://doi.org/10.1038/ng.592.

19. Wang S, Wu K, Yuan Q, Liu X, Liu Z, Lin X, et al. Control of grain size, shape and quality by OsSPL16 in rice. Nat Genet. 2012;44(8):950-4. https://doi. org/10.1038/ng.2327.

20. Shao $Y$, Zhou HZ, Wu Y, Zhang H, Lin J, Jiang $X$, et al. OsSPL3, an SBPdomain protein, regulates crown root development in Rice. Plant Cell. 2019; 31(6):1257-75. https://doi.org/10.1105/tpc.19.00038.

21. Chuck GS, Brown PJ, Meeley R, Hake S. Maize SBP-box transcription factors unbranched 2 and unbranched 3 affect yield traits by regulating the rate of lateral primordia initiation. Proc Natl Acad Sci U S A. 2014;111(52):18775-80. https://doi.org/10.1073/pnas.1407401112.

22. Xie K, Wu C, Xiong L. Genomic organization, differential expression, and interaction of SQUAMOSA promoter-binding-like transcription factors and microRNA156 in rice. Plant Physiol. 2006;142(1):280-93. https://doi.org/10.11 04/pp.106.084475.

23. Si L, Chen J, Huang X, Gong H, Luo J, Hou Q, et al. OsSPL13 controls grain size in cultivated rice. Nat Genet. 2016;48(4):447-56. https://doi.org/10.1038/ ng.3518.

24. Gou J, Fu C, Liu S, Tang C, Debnath S, Flanagan A, et al. The miR156-SPL4 module predominantly regulates aerial axillary bud formation and controls shoot architecture. New Phytol. 2017;216(3):829-40. https://doi.org/10.1111/ nph.14758.

25. Zhang J, Arro J, Chen Y, Ming R. Haplotype analysis of sucrose synthase gene family in three Saccharum species. BMC Genomics. 2013;14(1):314. https://doi.org/10.1186/1471-2164-14-314.

26. Henry RJ, Henry R, Kole CJG, genomics, sugarcane bo. Basic information on the sugarcane plant; 2010. p. 1-7.

27. D'Hont A. Unraveling the genome structure of polyploids using FISH and GISH; examples of sugarcane and banana. Cytogenet Genome Res. 2005; 109(1-3):27-33. https://doi.org/10.1159/000082378.

28. Zhang J, Zhang X, Tang H, Zhang Q, Hua X, Ma X, et al. Allele-defined genome of the autopolyploid sugarcane Saccharum spontaneum L. Nat Genet. 2018;50(11):1565-73. https://doi.org/10.1038/s41588-018-0237-2.

29. Wang LP, Jackson PA, Lu X, Fan YH, Foreman JW, Chen XK, et al. Evaluation of sugarcane $x$ Saccharum spontaneum progeny for biomass composition and yield components. Crop Sci. 2008;48(3):951-61. https://doi.org/10.2135/ cropsci2007.10.0555

30. Hoang NV, Furtado A, Mason PJ, Marquardt A, Kasirajan L, Thirugnanasambandam PP, et al. A survey of the complex transcriptome from the highly polyploid sugarcane genome using full-length isoform sequencing and de novo assembly from short read sequencing. BMC Genomics. 2017;18(1). https://doi.org/10.1186/s12864-017-3757-8.

31. Holub EBJNRG. The arms race is ancient history in Arabidopsis, the wildflower. Nat Rev Genet. 2001;2(7):516-27.
32. Yang Z, Bielawski JP. Statistical methods for detecting molecular adaptation. Trends Ecol Evol. 2000;15(12):496-503. https://doi.org/10.1016/S0169-534 7(00)01994-7.

33. Feng $X$, Wang $Y$, Zhang $N$, Zhang $X$, Wu J, Huang $Y$, et al. Systematic identification, evolution and expression analysis of the SPL gene family in sugarcane (Saccharum spontaneum). Trop Plant Biol. 2021;14(3):313-28. https://doi.org/10.1007/s12042-021-09293-4.

34. Liu M, Sun W, Ma Z, Huang L, Wu Q, Tang Z, et al. Genome-wide identification of the SPL gene family in Tartary Buckwheat (Fagopyrum tataricum) and expression analysis during fruit development stages. BMC Plant Biol. 2019;19(1):299. https://doi.org/10.1186/s12870-019-1916-6.

35. Du H, Feng BR, Yang SS, Huang YB, Tang YX. The R2R3-MYB transcription factor gene family in maize. PLoS One. 2012;7(6):e37463. https://doi.org/1 0.1371/journal.pone.0037463.

36. Chao LM, Liu YQ, Chen DY, Xue XY, Mao YB, Chen XY. Arabidopsis transcription factors SPL1 and SPL12 confer plant thermotolerance at reproductive stage. Mol Plant. 2017;10(5):735-48. https://doi.org/10.1016/j. molp.2017.03.010.

37. Xu M, Hu T, Zhao J, Park MY, Earley KW, Wu G, et al. Developmental functions of miR156-regulated SQUAMOSA PROMOTER BINDING PROTEINLIKE (SPL) genes in Arabidopsis thaliana. PLoS Genet. 2016;12(8):e1006263. https://doi.org/10.1371/journal.pgen.1006263.

38. Zhu T, Liu Y, Ma L, Wang X, Zhang D, Han Y, et al. Genome-wide identification, phylogeny and expression analysis of the SPL gene family in wheat. BMC Plant Biol. 2020;20(1):420. https://doi.org/10.1186/s12870-020-02 576-0.

39. Schwab R, Palatnik JF, Riester M, Schommer C, Schmid M, Weigel D. Specific effects of microRNAs on the plant transcriptome. Dev Cell. 2005;8(4):517-27. https://doi.org/10.1016/j.devcel.2005.01.018.

40. Goodstein DM, Shu S, Howson R, Neupane R, Hayes RD, Fazo J, et al. Phytozome: a comparative platform for green plant genomics. Nucleic Acids Res. 2012;40(Database issue):D1178-86. https://doi.org/10.1093/nar/ gkr944.

41. El-Gebali S, Mistry J, Bateman A, Eddy SR, Luciani A, Potter SC, et al. The Pfam protein families database in 2019. Nucleic Acids Res. 2019;47(D1): D427-32. https://doi.org/10.1093/nar/gky995.

42. Artimo P, Jonnalagedda M, Arnold K, Baratin D, Csardi G, de Castro E, et al. ExPASy: SIB bioinformatics resource portal. Nucleic Acids Res. 2012;40(Web Server issue):W597-603.

43. Hu B, Jin J, Guo AY, Zhang H, Luo J, Gao G. GSDS 2.0: an upgraded gene feature visualization server. Bioinformatics. 2015;31(8):1296-7. https://doi. org/10.1093/bioinformatics/btu817.

44. Wang $Y$, Tang H, Debarry JD, Tan X, Li J, Wang $X$, et al. MCScanX: a toolkit for detection and evolutionary analysis of gene synteny and collinearity. Nucleic Acids Res. 2012;40(7):e49. https://doi.org/10.1093/na r/gkr1293.

45. Krzywinski M, Schein J, Birol I, Connors J, Gascoyne R, Horsman D, et al. Circos: an information aesthetic for comparative genomics. Genome Res. 2009;19(9):1639-45. https://doi.org/10.1101/gr.092759.109.

46. Tamura K, Stecher G, Peterson D, Filipski A, Kumar S. MEGA6: molecular evolutionary genetics analysis version 6.0. Mol Biol Evol. 2013;30(12):2725-9. https://doi.org/10.1093/molbev/mst197.

47. Letunic I, Bork P. Interactive tree of life (iTOL) v3: an online tool for the display and annotation of phylogenetic and other trees. Nucleic Acids Res. 2016:44(W1):W242-5. https://doi.org/10.1093/nar/gkw290.

48. Letunic I, Bork P. Interactive Tree Of Life (iTOL) v4: recent updates and new developments. Nucleic Acids Res. 2019;47(W1):W256-9. https://doi.org/10.1 093/nar/gkz239.

49. Letunic I, Bork P. Interactive Tree Of Life (iTOL): an online tool for phylogenetic tree display and annotation. Bioinformatics. 2007;23(1):127-8. https://doi.org/10.1093/bioinformatics/btl529.

50. Bailey TL, Boden M, Buske FA, Frith M, Grant CE, Clementi L, et al. MEME SUITE: tools for motif discovery and searching. Nucleic Acids Res. 2009; 37(Web Server issue):W202-8.

51. Chen C, Chen H, Zhang Y, Thomas HR, Frank MH, He Y, et al. TBtools: an integrative toolkit developed for interactive analyses of big biological data. Mol Plant. 2020;13(8):1194-202. https://doi.org/10.1016/j.molp.2020.06.009.

52. Zhang Q, Hu W, Zhu F, Wang L, Yu Q, Ming R, et al. Structure, phylogeny, allelic haplotypes and expression of sucrose transporter gene families in Saccharum. BMC Genomics. 2016;17(1):88. https://doi.org/10.1186/s12864-01 6-2419-6. 
53. Chen Y, Zhang Q, Hu W, Zhang X, Wang L, Hua X, et al. Evolution and expression of the fructokinase gene family in Saccharum. BMC Genomics. 2017;18(1):197. https://doi.org/10.1186/s12864-017-3535-7.

54. Kim D, Paggi JM, Park C, Bennett C, Salzberg SL. Graph-based genome alignment and genotyping with HISAT2 and HISAT-genotype. Nat Biotechnol. 2019;37(8):907-15. https://doi.org/10.1038/s41587-019-0201-4

55. Trapnell C, Roberts A, Goff L, Pertea G, Kim D, Kelley DR, et al. Differential gene and transcript expression analysis of RNA-seq experiments with TopHat and Cufflinks. Nat Protoc. 2012;7(3):562-78. https://doi.org/10.1038/ nprot.2012.016.

\section{Publisher's Note}

Springer Nature remains neutral with regard to jurisdictional claims in published maps and institutional affiliations.

Ready to submit your research? Choose BMC and benefit from:

- fast, convenient online submission

- thorough peer review by experienced researchers in your field

- rapid publication on acceptance

- support for research data, including large and complex data types

- gold Open Access which fosters wider collaboration and increased citations

- maximum visibility for your research: over $100 \mathrm{M}$ website views per year

At $\mathrm{BMC}$, research is always in progress.

Learn more biomedcentral.com/submissions 